\title{
Developmentally regulated association of plastid division protein FtsZ1 to thylakoid membranes in Arabidopsis thaliana
}

\author{
El-Sayed EL-KAFAFI ${ }^{* 1,2}$, Mohamed KARAMOKO* ${ }^{*}$, Isabelle PIGNOT- \\ PAINTRAND $\dagger$, Didier GRUNWALD $\ddagger$, Paul MANDARON*, Silva LERBS-MACHE* \\ and Denis FALCONET**3
}

*Laboratoire Plastes et Différenciation Cellulaire, Université Joseph Fourier and Centre National de la Recherche Scientifique, BP 53, F-38041 Grenoble Cedex 9, France, †CERMAV, UPR 5301-CNRS, BP 53, F-38041 Grenoble Cedex 9, France, and ‡Département de Réponse et Dynamique Cellulaire, CEA, F 38054 Grenoble Cedex 9, France

${ }^{1}$ These authors contributed equally to this work.

${ }^{2}$ Present address: Institut de Biologie Intégrative des Plantes (IBIP), UMR 5004 AgroM/CNRS/INRA/UM2, Place Viala, 34060 Montpellier Cedex 1 France.

${ }^{3}$ To whom correspondence should be addressed (e-mail denis.falconet@ujfgrenoble.fr).

Short title: Association of plastid division protein FtsZ1 to thylakoids

Abbreviations used: IEP37, an inner envelope protein of $37 \mathrm{kDa}$; KARI, ketol-acid reducto-isomerase; OEP21, an outer envelope protein of $21 \mathrm{kDa}$; PSII, photosystem II; TEM, transmission electron microscopy; WT, wild-type. 


\section{SYNOPSIS}

FtsZ is a key protein involved in bacterial and organellar division. Bacteria have only one $f$ ts $Z$ gene, while chlorophytes (higher plants and green alga) have two distinct Fts $Z$ gene families, named FtsZ1 and FtsZ2. This raises the question why chloroplasts in these organisms need distinct FtsZ proteins to divide. In order to unravel new functions associated with FtsZ proteins, we have identified and characterized an Arabidopsis thaliana FtsZ1 loss-of-function mutant. ftsZ1 knockout mutants are impeded in chloroplast division and division is restored when FtsZ1 is expressed at low level. FtsZ1 overexpressing plants show a drastic inhibition in chloroplast division. Chloroplast morphology is altered in ftsZ1 with chloroplasts having abnormalities in the thylakoid membrane network. Overexpression of FtsZ1 also induced defects in thylakoid organization with an increased network of twisting thylakoids and larger grana. We show that FtsZ1, in addition to be present in the stroma, is tightly associated with the thylakoid fraction. This association is developmentally regulated since FtsZ1 is found in the thylakoid fraction of young developing plant leaves but not in mature and old plant leaves. Our results suggest that plastid division FtsZ1 may play a function during leaf development in thylakoid organization thus highlighting new functions for green plastid FtsZ.

Key words: Arabidopsis thaliana, plastid division, plastid localization, membrane dynamics, thylakoids, FtsZ 


\section{INTRODUCTION}

FtsZ plays an essential role in bacterial and archaeal division [1-3], in chloroplast division [4-6] and, in some lower organisms (algae, Slime molds), in mitochondrial division $[4,7]$. FtsZ, the most conserved of the bacterial cell division proteins, shares structural homology to the eukaryotic tubulin element and may be a progenitor of tubulin [8]. Bacterial FtsZ polymerizes like eukaryotic cytoskeletal proteins and forms the so-called $\mathrm{Z}$ ring, which forms a scaffold for assembly of at least ten other division proteins [9]. The $\mathrm{Z}$ ring adheres to the inside of the bacterial membrane and constricts to form a preseptal ingrowth, which then invaginates further to mediate division. Proteins involved in the cell division complex or divisome, are cytoplasmic, periplasmic and membrane-embedded proteins. No outer membrane protein has so far been linked to cell division.

While most bacteria (including cyanobacteria) have only one FtsZ protein, plastid FtsZ protein sequences form three clades, including green-plastid FtsZ1, green-plastid FtsZ2 and the red and chromophyte algal group [10,11]. Phylogenetic analysis suggests that $f t s Z$ gene duplication occurred subsequent to the endosymbiotic event. In chlorophytes, the gene duplication giving rise to the FtsZ1 and FtsZ2 gene families occurred before green algae branched from the ancestor of land plants [10-12]. The duplication event raises the question of why plastids in these organisms need two different proteins in order to divide when the ancestral bacteria use only one protein.

The major difference between FtsZ1 and FtsZ2 in both chlorophytes and nonchlorophytes lies within their C-terminal sequences. The C-terminal conserved motif, present in bacterial FtsZ, is required for direct interaction with ZipA and FtsA in Escherichia coli [13,14]. This domain is present in FtsZ2 but not in FtsZ1 in chlorophytes and in related sequences in nonchlorophytes. Homologues of ZipA and FtsA have not been identified in eukaryotic genomes, but the protein ARC6 in Arabidopsis shares homology with the cyanobacterial protein Ftn2 [15] and interacts with FtsZ2 via the C-terminal core domain [16] providing in planta evidence for a functional difference between the two FtsZ protein families in plants. The conserved Nterminal sequence is sufficient for bacterial FtsZ polymerization [17]. The major 
difference between FtsZ1 and FtsZ2 in higher plants concerns a single amino acid change in the conserved "tubulin signature motif" [18].

In addition, FtsZ1 and FtsZ2 differ in their biochemical properties and sub-plastidial localization [19]. Expression of FtsZ1 and FtsZ2 in E. coli differentially affects division and these different effects are related to the FtsZ2 C-terminal sequence. Only FtsZ1 is able to polymerize in vitro and forms GTP-dependent rod-shaped polymers and rings similar to the bacterial structures, but FtsZ2 can promote GTP-independent FtsZ1 polymerization. These data together with other results showing the interaction of only FtsZ2 with ARC6 and an earlier expression of FtsZ2 during the cell cycle in BY2 cells [18] suggests that FtsZ2 and FtsZ1 fulfil different functions during chloroplast division [19].

In addition to being a key element in the chloroplast division machinery, plant FtsZ proteins may be involved either directly or indirectly in the coordination of cell division and plastid division. An FtsZ1 isoform in the moss Physcomitrella patens is localized both in chloroplasts and in the cytoplasm, assembling into rings in both cell compartments and transfected cells expressing high amounts of the protein were impeded in cell division [20]. In higher plants, expression of both, FtsZ1 and FtsZ2, genes seems to be cell cycle regulated [18] and in Arabidopsis, AtCDT1 proteins are involved not only in nuclear DNA replication but also in plastid division by means of an interaction with ARC6 [21].

Here, we describe the characterization of an Arabidopsis ftsZ1 mutant in which chloroplast division is strongly impeded but restored when FtsZ1 is expressed at a low level. FtsZ1 overexpression in both backgrounds ( $f$ ts $Z 1$ and wild-type) induces a strong phenotype with a drastic effect on chloroplast morphology and division. Both null and FtsZ1 overexpressing plants have an accelerated development. fts $Z 1$-deficient plants have an altered thylakoid membrane network whereas FtsZ1 overexpression results in an increased network of twisting thylakoids and larger grana. We show that FtsZ1, in addition to being present in the stroma, is also found tightly associated with the thylakoid membranes and this association is developmentally regulated. 


\section{EXPERIMENTAL}

\section{Plant material, transformation and growth}

Arabidopsis, Wassilewskija ecotype (Ws), was used as wild-type (WT) plants for all experiments. The FtsZ1 T-DNA insertion mutant FST ${ }^{\circ} 128 \mathrm{~A} 08$ was obtained from the Flagdb/FST initiative (http://193.51.165.9/projects/FLAGdb++/HTML/index.shtml). Genomic DNA was amplified with the Tag6 (5'CTTTCATCTACGGCAATGTACCAGC-3') and Tag7 (5'-GTCGATAAGAAAAGGCAATTTGTAG-3') specific primers to detect the T-DNA. To follow the presence of the endogenous copy of the FtsZ1 gene and identify homozygous lines, genomic DNA was PCR-amplified with specific couples of primers: FtsZ1/D-FtsZ1/R and FtsZ1D-Tag6 (FtsZ1/D: ATGGCGATAATTCCGTTAGCACA-3'; FtsZ1/R:

5'-AGGGGCATCTGAAAAGAAGAT-3'). Homozygous lines were propagated by repeated self-pollination and the absence of FtsZ1 expression was assayed by immunoblotting with polyclonal FtsZ1 antibodies [18]. In order to exclude the presence of more than one T-DNA, Southern blot analysis was performed. Nuclear DNA of the selected lines was digested with EcoRI, a restriction enzyme with a unique site in FtsZ1 (within intron 1) and in the T-DNA. DNA fragments were fractionated on $0.8 \%$ agarose gels, denatured, and transferred onto nitrocellulose membrane. The membrane was hybridized, under standard high-stringency, with a T-DNA internal probe. For complementation analysis, the Arabidopsis FtsZ1 cDNA was ligated into the pFP101 vector that allows for the selection of transgenic Arabidopsis seeds via the GFP expression driven by the At2S3 seed-specific promoter [22]. The construct was expressed in the FST $\mathrm{N}^{\circ} 128 \mathrm{~A} 08$ mutant as well as in the WT plant. T1 plants were analyzed by immunoblotting with polyclonal FtsZ1 antibodies and by microscopy.

For plant growth, seeds were stratified at $4{ }^{\circ} \mathrm{C}$ for 2 days before growth on soil at $23^{\circ} \mathrm{C}$ with a $16 / 8$ hours light/dark photoperiod at a light intensity of $60 \mu \mathrm{mol} \mathrm{m} \mathrm{m}^{-2}$.

Protein isolation, chloroplast protein fractionation, treatments and immunoblotting analyses 
Total plant protein extracts were obtained following the Tanaka method [23]. Stromal, thylakoid and envelope proteins purified from lysed chloroplasts were separated on a step gradient of 0.93 and $0.6 \mathrm{M}$ sucrose in $10 \mathrm{mM}$ MOPS (pH 7.6) and $1 \mathrm{mM} \mathrm{MgCl}_{2}$ by centrifugation as described [24]. For high-salt, alkaline and ETDA washes, the purified thylakoid fraction (30 $\mu \mathrm{g}$ of protein) was incubated for $30 \mathrm{~min}$ at $0^{\circ} \mathrm{C}$ in $1.0 \mathrm{M} \mathrm{NaCl}$, $0.1 \mathrm{M} \mathrm{Na}_{2} \mathrm{CO}_{3}, \mathrm{pH} 11.5,0.1 \mathrm{~N} \mathrm{NaOH}$ or $5 \mathrm{mM}$ EDTA. The mixtures were then centrifuged and the pellets were resuspended in SDS loading buffer. For protease treatment, thylakoids were incubated for $30 \mathrm{~min}$ at $22^{\circ} \mathrm{C}$ with $100 \mu \mathrm{g} \mathrm{ml} l^{-1}$ thermolysin (SIGMA) in absence or presence of $5 \mathrm{mM}$ EGTA. Proteolysis was stopped by supplementing the assays with $20 \mathrm{mM}$ EGTA, thylakoids were re-isolated by centrifugation, washed once and suspended in SDS loading buffer.

Protein concentrations were determined by using the BIO-RAD DC protein and BSA as standard. When necessary, Coomassie-stained gels were used to calibrate loading. For immunoblot analyses, the proteins were separated by SDS-PAGE, blotted to Immobilon-P membranes (MILLIPORE) and incubated with antibodies as described previously [19].

\section{Pigment analyses and chlorophyll fluorescence}

For pigment analyses, leaf samples ( $70 \mathrm{mg}$ fresh weight) were frozen in liquid nitrogen prior to grinding in N,N-dimethylformamide (DMF). The resulting extracts, after bubbling with nitrogen, were stored in the dark for $48 \mathrm{~h}$ at $4{ }^{\circ} \mathrm{C}$. Extracts were centrifuged for $5 \mathrm{~min}$ at $12000 \mathrm{~g}$ and pigment analyses were performed by HPLC using a $5 \mathrm{~mm}$ reverse-phase $\mathrm{C}_{30}$ column $(250 \mathrm{X} 4 \mathrm{~mm})$ coupled to a $20 \mathrm{X} 4.6 \mathrm{~mm} \mathrm{C}_{30}$ guard (YMC, Inc., USA) and a ProStar 330 photodiode array detector (VARIAN ANALYTICAL INSTRUMENTS, USA), as described previously [25]. Peak areas of the standards were determined using the Varian Poly View 2000 software supplied.

The maximal quantum yield of PSII photochemistry (F) with an Fv/Fmax ratio was determined on attached leaves at room temperature by chlorophyll fluorometry using a PAM-2000 modulated fluorometer (WALTZ, Effeltrich, Germany) as described [26]. 


\section{Confocal microscopy, transmission electron microscopy and immunogold electron microscopy}

Chloroplasts in mesophyll and shoot cells were analyzed by imaging chlorophyll fluorescence by confocal laser-scanning microscopy with a Leica TCS-SP2 operating system (LEICA, Germany). Chlorophyll was excited at $633 \mathrm{~nm}$ and the emitted fluorescence was detected between $643 \mathrm{~nm}$ and $720 \mathrm{~nm}$.

For transmission electron microscopy (TEM) and immunolocalization, leaf samples of three-week-old plants were fixed in $2.5 \%$ (v/v) glutaraldehyde in $0.1 \mathrm{M}$ cacodylate buffer for $2 \mathrm{~h}$ at room temperature. The samples were subsequently washed and postfixed with $1 \%$ osmium tetraoxide in water for $1 \mathrm{~h}$ at $4^{\circ} \mathrm{C}$ and then dehydrated in ethanol and infiltrated with ethanol/resin mixture (2/3-1/3, 1/3-2/3 for Epon and 80-20, 60-40, 40-60, 20-80, 100, 100 last infiltration overnight for LR-White). Tissues were embedded in Epon for structural study and in LR-White for immunolocalization. Ultrathin sections $(60 \mathrm{~nm})$ were prepared with a diamond knife on an UC6 Leica ultramicrotome and collected on formvar-coated $200 \mu \mathrm{m}$ mesh nickel grids. Ultrathin sections were post-stained with 5\% uranyl acetate in water and lead citrate before examining on a Philips CM200 electron microscope (PHILIPS, Netherlands).

For immunolocalization, the sections were first incubated with $5 \%(\mathrm{w} / \mathrm{v})$ BSA in PBS for $1 \mathrm{~h}$ at room temperature and then with anti-FtsZ1 antibodies (dilution 1:4 in 1\% BSA in PBS) overnight at $4^{\circ} \mathrm{C}$. Samples were then incubated with goat anti-rabbit $\operatorname{IgG}$ conjugated to $10 \mathrm{~nm}$ gold particles at a dilution of 1:20 in 1\% BSA in PBS for $1 \mathrm{~h}$ at room temperature. Controls were performed by excluding the primary antibody. Finally, sections were post-stained with 5\% uranyl acetate in water and lead citrate.

\section{RESULTS}

\section{Phenotypic characterization of $f$ ts $Z 1$-deficient and FtsZ1 overexpressing plants}

The Arabidopsis genome encodes a single FtsZ1 plastid division protein on chromosome 5 at the locus At5g55280. One Arabidopsis line containing a T-DNA insertion in exon 4 was identified by the Genoplante FLAGdb/FST initiative (Figure 
1A). Plants homozygous for the mutant alleles were identified by PCR analysis of segregating plants (Figures $1 \mathrm{~B}$ and $1 \mathrm{C}$ ). Segregation values observed for kanamycin resistance (three-fourth resistant and one-fourth sensitive individuals after selfpollination of heterozygous plants) indicated that the mutant lines contained only one insertion of the T-DNA in their nuclear genome. The presence of only one T-DNA was also confirmed by Southern blot analysis (Figure 1D). Only the $4.8 \mathrm{Kbp}$ EcoRI fragment, expected from the restriction data, was detected. Western blot analysis confirmed the absence of FtsZ1 protein in the selected mutant lines (Figure 1E). Western blots showed FtsZ1 protein expression in WT plants and in the heterozygous line 1 , but no expression in the homozygous lines 2, 3 and 4 .

Mature ftsZ1 mesophyll and stem cells contained fewer, but larger chloroplasts than the corresponding cells in WT, although the cell sizes between the two plants were similar (Figure 2A, upper panel and Figure 2B). The average chloroplast number at the equatorial plan decreased from 16 in WT mesophyll cells and 14 in WT stem cells to 7 and 4 , respectively, in $f t s Z 1$ cells. The mutant phenotype was rescued by low-level expression of the FtsZ1 cDNA as shown by Western blotting, which did not alter the WT phenotype when expressed in WT plants (Figure 2A, middle panel). However, overexpression of FtsZ1 had a drastic effect on chloroplast division in both the WT and ftsZ1 backgrounds with cells harbouring only few enlarged chloroplasts (Figure 2A, lower panel). This corroborates previous results showing altered stoichiometry of FtsZ affecting chloroplast division [27]. As illustrated with the stem cells (Figure 2B), all cells of a given tissue were strongly affected by the absence or overexpression of the FtsZ1 protein. In all the mutant cells examined the shape of the large chloroplasts was irregular having lost the characteristic globular structure as observed in the WT plants. Compared with WT plants, ftsZ1 and FtsZ1 overexpressing lines exhibited accelerated growth (Figure 2C) and began flowering about one week earlier, on average, than WT plants (two weeks after germination for the mutant plants versus three weeks for the WT plants). ftsZ1 and 35S::FtsZ1 plants flowered with the same number of rosette leaves (with an average of 8 leaves) when the WT plants flowered with an average of 14 larger rosette leaves. After six weeks of growth, the size of the mutant plants was greatly reduced compared to the WT plants. Both mutant lines were able to produce viable seeds. Photosynthesis was investigated using pulse-amplitude-modulated 
chlorophyll fluorescence analysis and pigment content on three week old plantlets. The ratio of variable fluorescence $(\mathrm{Fv})$ :maximum fluorescence $(\mathrm{Fm})$ was identical between the mutants plants and the WT plants (Figure 2D). This indicates that the photosystem II (PSII) reaction centre was properly assembled and photochemically competent in both mutant plants. Equally, the amount of the photosynthetic pigments was unaltered in both mutant plants (Figure 2E).

\section{Chloroplast morphology and ultrastructure is affected in ftsZ1 and 35S::FtsZ1 plants}

Three-week-old rosette leaves were fixed for TEM and observed at different levels of magnification. In WT plants, individual ellipsoidal chloroplasts, aligned along the cytoplasmic membrane, were observed at the cellular level (Figure 3A). These chloroplasts contained starch granules, and their internal structures, including evenly stacked granal thylakoids connected by stromal lamellae, were completely developed (Figures 3D and 3G). These lamellae are oriented along the convex side of the chloroplast facing the interior of the cell. In contrast, abnormally long chloroplasts were observed in the leaves of ftsZ1 (Figure 3B) and 35S::FtsZ1 plants (Figure 3C). Although the long chloroplasts in the null plant were aligned along the plasma membrane, as was observed in the WT plant, the chloroplasts were detached from the plasma membrane at several points in the FtsZ1 overexpressing plant. Changes in thylakoid orientation were often observed in the null plant (Figure 3E; see also supplementary Figure S1), and membranes appeared less appressed (Figure 3H). Overexpression of FtsZ1 induced an increased network of twisting thylakoids (Figure 3F) and an increased number of thylakoids per grana stack (Figure 3I). An increase in starch granule number was observed in the null mutants, but no starch granules were observed in FtsZ1 overexpressing plants.

\section{FtsZ1 is localized within the stroma, but also found associated with the thylakoids}

The changes in thylakoid organization in ftsZ1 null and overexpressing plants indicated a possible role of FtsZ1 in thylakoid integrity, and therefore suggested an association or 
at least some contact between FtsZ1 and thylakoid membranes. In order to test this hypothesis, we investigated the localization of FtsZ1 proteins in WT Arabidopsis plants by suborganellar fractionation and western blotting, using an anti-FtsZ1 antibody previously shown to react with proteins from purified chloroplast extracts [28] (Figure 4A). The stromal, thylakoid and envelope fractions were purified from lysed chloroplasts by sucrose gradient centrifugation [24]. Blots were first probed with the anti-FtsZ1 antibody, and subsequently probed with antisera against KARI (ketol-acid reducto-isomerase), a stromal protein involved in the amino acid biosynthetic pathway [29], PsbB (CP47), a thylakoid-integrated subunit of the photosystem II reaction center, IEP37, an inner envelope protein of $37 \mathrm{kDa}$ [30] and OEP21, a $21 \mathrm{kDa}$ protein of the outer envelope [31]. An FtsZ1 signal colocalized with the stromal and thylakoid controls, but not with the envelope proteins, suggesting that FtsZ1 localizes to thylakoid membranes (Figure 4A). Purified thylakoids were next washed with $1 \mathrm{M} \mathrm{NaCl}, 0.1 \mathrm{M}$ $\mathrm{Na}_{2} \mathrm{CO}_{3}, \mathrm{pH} 11.5$, or $0.1 \mathrm{M} \mathrm{NaOH}$ to further characterize the association of FtsZ1 with the thylakoid membranes (Figure 4B). FtsZ1 was not released from the thylakoids by ionic extraction but was solubilized by alkaline extractions indicating that it is peripherally associated with the membrane. The localization with the thylakoid membranes is independent of magnesium concentration because in presence of EDTA, FtsZ1 is still detected in the thylakoid fraction (Figure 4B). As a control, release of PsbB from membranes was tested under identical conditions, but none was observed. It is therefore possible that FtsZ1 is associated with one leaflet of the membrane via lipid interaction.

To determine FtsZ1 topology, thermolysin proteolysis was performed with isolated thylakoids (Figure 4C). Under such treatment, proteins on the stromal side of the thylakoid membrane are degradable, while lumen proteins, not accessible to thermolysin, are protected. FtsZ1 was accessible to thermolysin in the absence of EGTA, while the addition of EGTA inhibited the cleavage reaction, suggesting that FtsZ1 is located on the stromal-facing side of the thylakoid membranes.

To confirm the localization of FtsZ1 visually, immunoelectron microscopy was performed with three-week-old Arabidopsis WT and FtsZ1 overexpressing plants (35S::FtSZ1). Small clusters and large clusters of gold particles in the WT and 35S FtsZ1 plants, respectively, were observed in close association with the thylakoid 
network (Figure 5). The large number of gold particles in the $35 S:: F t s Z 1$ plant are in agreement with the results observed by immunoblot analysis (Figure 2A).

\section{Association of FtsZ1 with the thylakoids is developmentally regulated}

FtsZ1 was reported previously to be a soluble protein found only in the stromal fraction $[19,32]$, while our results suggest that FtsZ1 is also associated with thylakoid membranes. To understand this discrepancy, we investigated whether FtsZ1 association with the thylakoids differed at various points during plant development. To test this hypothesis we examined developmentally-associated changes in FtsZ1 protein amount and localization by western blotting. Proteins from subplastidial fractions were obtained from plants grown for 22, 35 and 43 days. A strong FtsZ1 signal was observed in the thylakoid fraction as well as in the stromal fraction of plants grown for 22 days (Figure 6). After 35 days of growth, only a faint signal was observed in the thylakoid fraction while a strong signal was observed in the stromal fraction. FtsZ1 is no longer detected in the thylakoid fraction, but still strongly present in the stroma in plants grown for 43 days. As expected, KARI was present only in the stroma, whereas PsbB was found exclusively in thylakoids indicating the purity of the two fractions. The correlation between the level of FtsZ1 in the thylakoid fraction and developmental stage of the tissue demonstrated the regulation of FtsZ1 localization within the chloroplast.

\section{DISCUSSION}

In order to highlight new plant-specific functions for FtsZ1, we have characterized a ftsZ1 null mutant of Arabidopsis. As expected from previous reports using an antisense strategy [27], fts Z1 mutant cells contained fewer but larger chloroplasts than WT plants. Inhibition of chloroplast division is rescued by expressing FtsZ1 in these mutant plants, but the complementation is dose dependent, since weak expression restores the WT phenotype while a strong expression causes inhibition of chloroplast division and changes in chloroplast morphology, as previously shown [33]. Such a dose dependent effect also occurs when FtsZ1 is expressed in E. coli mimicking the expression of other bacterial $f t s Z$ genes in E. coli [19]. These results confirm that a strict stoichiometry 
between subunits of the chloroplast division machinery is necessary for proper division. The overall life cycle of both $f t s Z 1$ plants and FtsZ1 overexpressing lines is not affected, confirming that impaired plastid division has no significant effect on plant growth and development [34], although these plants are smaller than WT controls, flower earlier and contain a reduced number of rosette leaves. This phenotype is not a direct consequence of smaller cells and/or diminished photosynthetic yield, since the cell size and photosynthetic capacity, as determined by measuring the maximal photochemical efficiency of PSII and quantifying the photosynthetic pigments, are unaltered in both transgenic lines. Developmental variation in the rate of leaf initiation has been suggested to result from change in the rate of cell division in the shoot apical meristem (SAM) [35]. The phenotype observed in the mutant plants might be driven by changes in cell division in the SAM thus connecting cell division and plastid division as suggested by different studies [18,20,21,36].

Mesophyll chloroplast ultrastructure is affected differently in the null and FtsZ1 overexpressing plants. FtsZ1 chloroplasts have frequent changes in their thylakoid orientation and fewer thylakoids per granal stack while FtsZ1 overexpressing chloroplasts display a highly disturbed thylakoid network with an increase in granal stacking. Organizational changes of the thylakoid membranes within some, but not all, chloroplast division mutants have also been described. Granal stacks in GCl-deficient giant chloroplasts are more densely packed than in WT plants [37]. Thylakoids in arc5 and arc6 chloroplasts are also highly stacked when compared to WT chloroplasts and show increased stacking when plants are grown under high light [38]. Under the same conditions, a decrease is observed with WT plants. Interestingly, FtsZ1 overexpressing plants show an increased network of twisting thylakoids similar to those observed in arc3 [38]. These results demonstrate that some proteins involved in plastid division also affect chloroplast internal structures. GC1 is plastid-localized and is anchored to the stromal surface of the chloroplast inner envelope [37]. Arc5 encodes a cytosolic dynamin-like protein and forms a cytosolic ring structure on the outside of the choroplast [39]. Arc6, which encodes a J-domain protein and is a homologue of the cyanobacterial cell division protein Ftn2 [15], has been shown to be an integral inner envelope membrane protein. Arc3, a fusion of FtsZ and phosphatidylinositol-4phosphate 5-kinase (PIP5K) [40] has recently been shown to be located in the stroma 
[41]. None of these proteins has been found associated with thylakoids, suggesting that the effect on the thylakoid network in these mutants is indirect. The twisting thylakoids observed in arc3, resembling those observed in the FtsZ1 overexpressing plants, might result from the misplacement of FtsZ1. As a matter of fact, Arc3 has been shown to interact specifically with FtsZ1, acting in division site placement [41].

The localization of FtsZ proteins in higher plants is more complex. Both, FtsZ1 and FtsZ2, have been found in the chloroplast stromal compartment in Arabidopsis chloroplasts [32], but localization in the thylakoid fraction has not been addressed previously. We have shown that FtsZ1 and FtsZ2 are localized within the stroma in chloroplasts from mature spinach (Spinacia oleracea) leaves, but that FtsZ2 is also found associated with the chloroplast envelopes [19]. In this report, we show that FtsZ1 is present in the stromal and thylakoid fractions from the chloroplasts of young Arabidopsis seedlings. Moreover, we demonstrate that the levels of FtsZ1 associated with the thylakoids decreases with the age of the plants. This result shows that FtsZ1 localization within the chloroplast is developmentally regulated and suggests that the protein fulfils specific functions at different stages of plant development. It is not known yet whether the difference in localization between spinach and Arabidopsis is a consequence of the different developmental stage and/or culture conditions of the analyzed plants. In the previous experiments, spinach leaves, purchased from a local market, were grown under external conditions when Arabidopsis plants in this study were grown under strict controlled temperature and light conditions. Whether FtsZ1 association with the thylakoids is dependent of these growth conditions is not known yet. Alternatively, it is possible that the discrepancy reflects species difference.

The thylakoid organization phenotypes associated with the absence or overexpression of FtsZ1 in the mutant plants corroborate the hypothesis that FtsZ1 is involved in determining thylakoid morphology. It is therefore conceivable that the organizational changes of the thylakoid membranes within the arc chloroplasts reflect an increased localization of FtsZ1 within the thylakoids in the absence of the ARC proteins. Interestingly, dynamin like proteins, which are involved in plastid division [39], have also been shown to be a determinant in thylakoid morphology [42, 43]. In Arabidopsis FZL, which is related to FZO, a dynamin protein involved in mitochondrial morphology in fungi and animals, is distributed between the thylakoid and envelope membranes. Fzl mutants have abnormalities in the morphology and distribution of 
granal and stromal thylakoids [43]. The results presented in this report suggest that the dynamic duo of FtsZ and dynamin, which has been shown to be involved in plastid division, participate in the remodeling of the thylakoid membranes. FtsZ1 and FZL are not involved in the early steps of thylakoid biogenesis since both $\mathrm{fts} Z 1$ and $f z o$ chloroplasts have a preformed thylakoid network. Other proteins such as VIPP1 [44] and Thf1 [45] have been implicated in chloroplast vesicle trafficking and shown to affect thylakoid formation. The association of FtsZ1 with the thylakoids during leaf expansion suggests that FtsZ1 might be involved in thylakoid membrane partitioning to daughter plastids during division and in the re-arrangement of the internal membrane structures when new thylakoid membranes are synthesized, thus highlighting specific functions for green plastid FtsZ.

\section{ACKNOWLEDGEMENTS}

We thank Catherine Albrieux (PCV, Grenoble) for help with the thylakoid purification; Maryse Block (PCV, Grenoble) for helpful comments concerning the chloroplast membrane system; Hélène Pesey, Jean-Pierre Alcaraz, Eliane Charpentier, Abder Lahroussi and Damien Nissou for technical assistance; Marcel Kuntz for help with the pigment analysis; Renaud Dumas (PCV, Grenoble) for the gift of the KARI antibody and Maryse Block (PCV, Grenoble) for IEP37 and OEP21 antibodies. We thank Livia Merendino and Stéphane Lobreaux for critical reading of the manuscript and Thomas Bollenbach (Cornell University) for advice on improving the English in the manuscript. This work was supported by a grant (ACI DRAB 03/41, N 03590 to D.F. and $\mathrm{N}^{\circ} 03592$ to I.P.P.) from the Centre National de la Recherche Scientifique (CNRS) and the Ministère de l'Education Nationale (MEN), by the Egyptian government (fellowship to E.S.E.K.) and by the Ivory Coast government (fellowship to M.K.).

\section{REFERENCES}

1 Addinall, S. G. and Holland, B. (2002) The tubulin ancester, FtsZ, draughtman, designer and driving force for bacterial cytokinesis. J. Mol. Biol. 318, 219-236 
2 Errington, J., Daniel, R. A. and Scheffers, D.-J. (2003) Cytokinesis in Bacteria. Microbiol. Mol. Biol. Rev. 67, 52-65

3 Margolin, W. (2003) Bacterial division: the fellowship of the ring. Curr. Biol. 13, R16-R18

4 Osteryoung, K. W. and Nunnari, J. (2003) The division of endosymbiotic organelles. Science 302, 1698-1704

5 Miyagishima, S., Nishida, K. and Kuroiwa, T. (2003) An evolutionary puzzle: chloroplast and mitochondrial division rings. Trends Plant Sci. 8, 432-438

6 Maple, J. and Moller, S. G. (2005) An emerging picture of plastid division in higher plants. Planta 223, 1-4

7 Gilson, P. R. and Beech, P. L. (2001) Cell division protein FtsZ: running rings around bacteria, chloroplasts and mitochondria, Res. Microbiol. 152, 3-10

8 Lowe, J. and Amos, L. A. (1998) Crystal structure of the bacterial cell division protein FtsZ. Nature 391, 203-206

9 Goehring, N. W. and Beckwith, J. (2005) Diverse paths to midcell: assembly of the bacterial cell division machinery. Curr. Biol. 15, R514-526

10 Stokes, K. D. and Osteryoung, K. W. (2003) Early divergence of the FtsZ1 and FtsZ2 plastid division gene families in photosynthetic eukaryotes. Gene 320, 97 108

11 Kiefel, B. R., Gilson, P. R. and Beech, P. L. (2004) Diverse eukaryotes have retained mitochondrial homologues of the bacterial division protein FtsZ. Protist 155, 105-115

12 Miyagishima, S. Y. (2005) Origin and evolution of the chloroplast division machinery. J. Plant Res. 118, 295-306

13 Ma, X. and Margolin, W. (1999) Genetic and functional analyses of the conserved C-terminal core domain of Escherichia coli FtsZ. J. Bacteriol. 181, 7531-7544

14 Osteryoung, K. W. and McAndrew, R. S. (2001) The plastid division machine. Annu. Rev. Plant Physiol. Plant Mol. Biol. 52, 315-333

15 Vitha, S., Froehlich, J. E., Koksharova, O., Pyke, K. A., van Erp, H. and Osteryoung, K. W. (2003) ARC6 is a J-domain plastid division protein and an evolutionary descendant of the cyanobacterial cell division protein Ftn2. Plant Cell 15, 1918-1933 
16 Maple, J., Aldridge, C. and Moller, S. G. (2005) Plastid division is mediated by combinatorial assembly of plastid division proteins. Plant J. 43, 811-823

17 Wang, X., Huang, J., Mukherjee, A., Cao, C. and Lutkenhaus, J. (1997) Analysis of the interaction of FtsZ with itself, GTP, and FtsA. J. Bacteriol. 179, 5551-5559

18 El-Shami, M., El-Kafafi, S., Falconet, D. and Lerbs-Mache, S. (2002) Cell cycledependent modulation of FtsZ expression in synchronized tobacco BY2 cells. Mol. Genet. Genomics 267, 254-261

19 El-Kafafi, S., Mukherjee, S., El-Shami, M., Putaux, J. L., Block, M. A., PignotPaintrand, I., Lerbs-Mache, S. and Falconet, D. (2005) The plastid division proteins, FtsZ1 and FtsZ2, differ in their biochemical properties and sub-plastidial localization. Biochem. J. 387, 669-676

20 Kiessling, J., Martin, A., Gremillon, L., Rensing, S. A., Nick, P., Sarnighausen, E., Decker, E. L. and Reski, R. (2004) Dual targeting of plastid division protein FtsZ to chloroplasts and the cytoplasm. EMBO Rep. 5, 889-894

21 Raynaud, C., Perennes, C., Reuzeau, C., Catrice, O., Brown, S. and Bergounioux, C. (2005) Cell and plastid division are coordinated through the prereplication factor AtCDT1. Proc. Natl. Acad. Sci. U.S.A. 102, 8216-8221

22 Bensmihen, S., To, A., Lambert, G., Kroj, T., Giraudat, J. and Parcy, F. (2004) Analysis of an activated ABI5 allele using a new selection method for transgenic Arabidopsis seeds. FEBS Lett. 561, 127-131

23 Hurkman, W.-J. and Tanaka, C. K. (1986) Solubilization of plant membaneproteins for analysis by two-dimensional gel electrophoresis. Plant Physiol. 81, 802-806

24 Block, M. A., Tewari, A. K., Albrieux, C., Marechal, E. and Joyard, J. (2002) The plant S-adenosyl-L-methionine:Mg-protoporphyrin IX methyltransferase is located in both envelope and thylakoid chloroplast membranes. Eur. J. Biochem. 269, 240-248

25 Fraser, P. D., Pinto, M. E., Holloway, D. E. and Bramley, P. M. (2000) Technical advance: application of high-performance liquid chromatography with photodiode array detection to the metabolic profiling of plant isoprenoids. Plant J. 24, 551-558

26 Klimyuk, V. I., Persello-Cartieaux, F., Havaux, M., Contard-David, P., Schuenemann, D., Meiherhoff, K., Gouet, P., Jones, J. D., Hoffman, N. E. and 
Nussaume, L. (1999) A chromodomain protein encoded by the Arabidopsis CAO gene is a plant-specific component of the chloroplast signal recognition particle pathway that is involved in LHCP targeting. Plant Cell 11, 87-99

27 Osteryoung, K. W., Stokes, K. D., Rutherford, S. M., Percival, A. L. and Lee, W. Y. (1998) Chloroplast division in higher plants requires members of two functionally divergent gene families with homology to bacterial ftsZ. Plant Cell 10, 1991-2004

28 Osteryoung, K. W. and Vierling, E. (1995) Conserved cell and organelle division. Nature 376, 473-474

29 Dumas, R., Biou, V., Halgand, F., Douce, R. and Duggleby, R. G. (2001) Enzymology, structure, and dynamics of acetohydroxy acid isomeroreductase. Acc. chem. Res. 34, 399-408

30 Block, M. A., Dorne, A. J., Joyard, J. and Douce, R. (1983) Preparation and characterization of membrane fractions enriched in outer and inner envelope membranes from spinach chloroplasts. I - Electrophoretic and Immunochemical Analyses. J. Biol. Chem. 258, 13273-13280

31 Jouhet, J., Marechal, E., Baldan, B., Bligny, R., Joyard, J. and Block, M. A. (2004) Phosphate deprivation induces transfer of DGDG galactolipid from chloroplast to mitochondria. J. Cell. Biol. 167, 863-874

32 McAndrew, R. S., Froehlich, J. E., Vitha, S., Stokes, K. D. and Osteryoung, K. W. (2001) Colocalization of plastid division proteins in the chloroplast stromal compartment establishes a new functional relationship between FtsZ1 and FtsZ2 in higher plants. Plant Physiol. 127, 1656-1666

33 Stokes, K. D., McAndrew, R. S., Figueroa, R., Vitha, S. and Osteryoung, K. W. (2000) Chloroplast division and morphology are differentially affected by overexpression of FtsZ1 and FtsZ2 genes in Arabidopsis. Plant Physiol. 124, $1668-1677$

34 Pyke, A. K. and Leech, M. R. (1994) A genetic analysis of chloroplast division and expansion in Arabidopsis thaliana. Plant Physiol. 104, 201-207

35 Poethig, R. S. (2003) Phase change and the regulation of developmental timing in plants. Science 301, 334-336 
36 Asano, T., Yoshioka, Y., Kurei, S., Sakamoto, W. and Machida, Y. (2004) A mutation of the CRUMPLED LEAF gene that encodes a protein localized in the outer envelope membrane of plastids affects the pattern of cell division, cell differentiation, and plastid division in Arabidopsis. Plant J. 38, 448-459

37 Maple, J., Fujiwara, M. T., Kitahata, N., Lawson, T., Baker, N. R., Yoshida, S. and Moller, S. G. (2004) GIANT CHLOROPLAST 1 is essential for correct plastid division in Arabidopsis. Curr. Biol. 14, 776-781

38 Ii, J. A. and Webber, A. N. (2005) Photosynthesis in Arabidopsis thaliana mutants with reduced chloroplast number. Photosynth. Res. 85, 373-384

39 Gao, H., Kadirjan-Kalbach, D., Froehlich, J. E. and Osteryoung, K. W. (2003) ARC5, a cytosolic dynamin-like protein from plants, is part of the chloroplast division machinery. Proc. Natl. Acad. Sci. U.S.A. 100, 4328-4333

40 Shimada, H., Koizumi, M., Kuroki, K., Mochizuki, M., Fujimoto, H., Ohta, H., Masuda, T. and Takamiya, K. (2004) ARC3, a chloroplast division factor, is a chimera of prokaryotic FtsZ and part of eukaryotic phosphatidylinositol-4phosphate 5-kinase. Plant Cell Physiol. 45, 960-967

41 Maple, J., Vojta, L., Soll, J. and Moller, S. G. (2007) ARC3 is a stromal Z-ring accessory protein essential for plastid division. EMBO Rep. 8, 293-299

42 Park, J. M., Cho, J. H., Kang, S. G., Jang, H. J., Pih, K. T., Piao, H. L., Cho, M. J. and Hwang, I. (1998) A dynamin-like protein in Arabidopsis thaliana is involved in biogenesis of thylakoid membranes. EMBO J. 17, 859-867

43 Gao, H., Sage, T. L. and Osteryoung, K. W. (2006) FZL, an FZO-like protein in plants, is a determinant of thylakoid and chloroplast morphology. Proc. Natl. Acad. Sci. U.S.A. 103, 6759-6764

44 Kroll, D., Meierhoff, K., Bechtold, N., Kinoshita, M., Westphal, S., Vothknecht, U. C., Soll, J. and Westhoff, P. (2001) VIPP1, a nuclear gene of Arabidopsis thaliana essential for thylakoid membrane formation. Proc. Natl. Acad. Sci. U.S A. 98, 4238-4242

45 Wang, Q., Sullivan, R. W., Kight, A., Henry, R. L., Huang, J., Jones, A. M. and Korth, K. L. (2004) Deletion of the chloroplast-localized Thylakoid formation1 gene product in Arabidopsis leads to deficient thylakoid formation and variegated leaves. Plant Physiol. 136, 3594-3604 


\section{FIGURE LEGENDS}

\section{Figure 1 fts $Z 1$ characterization}

(A) Gene structure of FtsZ1. Black boxes, exons; lines, introns. The position of the TDNA insertion in exon 4 is indicated together with the primer locations. (B) and $(\mathbf{C})$ Identification of homozygous lines. PCR amplification with the indicated primers on genomic DNA extracted from WT and selected lines (1-4). M: DNA size marker. (D) T-DNA insertion number in the mutant lines as analyzed by Southern blot with the T-DNA internal probe. (E) Expression of FtsZ1 in the selected plants as analyzed by immunobloting with FtsZ1 antiserum.

\section{Figure 2 Phenotype of $f$ ts $Z 1$ and FtsZ1 overexpressing plants}

(A) Upper panels: confocal micrographs of chloroplasts of WT (left column) and ftsZ1 (right column) plant leaves; middle and lower panels: WT and ftsZ1 plants expressing FtsZ1 under the control of the 35S promoter. Relative FtsZ1 expression levels are shown by immonoblots on the left side of each panel. Bar: $20 \mu \mathrm{m}$. (B) Confocal micrographs of hypocotyl cells from WT, ftsZ1 and overexpressing (35S::FtsZ1) plants. Bar: $40 \mu \mathrm{m}$. (C) Phenotype of four-week-old WT, ftsZ1 and 35S::FtsZ1 plants. (D) Chlorophyll fluorescence was measured as described in Experimental. Error bars represent the standard deviation from three measurements. (E) Accumulation of pigments indicated on the horizonal axis was measured as described in Experimental. Error bars represent the standard deviation from three measurements.

\section{Figure 3 FtsZ1 affects chloroplast morphology and ultrastructure}

Electron micrographs of rosette leaf mesophyll chloroplasts from three-week-old WT, FtsZ1 and 35S::FtsZ1 plants. (A-C) Overviews showing the chloroplast number and morphology within the cells (bar: $5 \mu \mathrm{m}$ ). (D-F) Inside chloroplast views showing the thylakoid arrangement (bar: $1 \mu \mathrm{m}$ ). (G-I) Higher magnification showing the grana stacking (bar: $200 \mathrm{~nm}$ ). 


\section{Figure 4 FtsZ1 is localized in the stroma and thylakoid membranes}

(A) Immunoblots of protein fractions (30 $\mu \mathrm{g}$ of each) isolated from chloroplasts, stroma, thylakoids and envelopes of four-week-old plants were analyzed with FtsZ1, KARI, PsbB (AGRISERA, Sweden), IEP37 and OEP21 antisera. The faint signal in the thylakoid fraction observed with the envelope markers indicated a very low level of contamination of the thylakoids with envelope membranes. (B) Purified thylakoids (30 $\mu \mathrm{g}$ of proteins) were incubated for $30 \mathrm{~min}$ in the indicated solutions and the proteins present after centrifugation in the pellet were analyzed by immunoblotting with either the FtsZ1 or PsbB antibodies. (C) Thylakoid fractions were incubated with thermolysin $\left(100 \mu \mathrm{g} \mathrm{ml}^{-1}\right)$ in absence (-EGTA) or presence (+EGTA) of EGTA. The control (no thermolysin) proteins (thylakoid) and treated thylakoid proteins were analyzed by immunoblotting with the FtsZ1 antibody.

Figure 5 Immunolocalization of the FtsZ1 protein in association with Arabidopsis thylakoids

Ultrathin sections of chloroplasts from WT and overexpressing (35S::FtsZ1) plants were incubated successively with anti-FtsZ1 antibodies and goat anti-(rabbit IgG) secondary antibody conjugated to colloidal gold. Gold clusters in association with the thylakoids are indicated by the arrowheads in both panels, (bar: $100 \mathrm{~nm}$ ).

Figure 6 FtsZ1 association with the thylakoids is developmentally regulated

Immunoblot analysis of protein fractions (30 $\mu \mathrm{g}$ of each) isolated from chloroplasts, stroma and thylakoids of 22-, 35- and 43-day-old plants, respectively, with FtsZ1, KARI and PsbB antisera. 

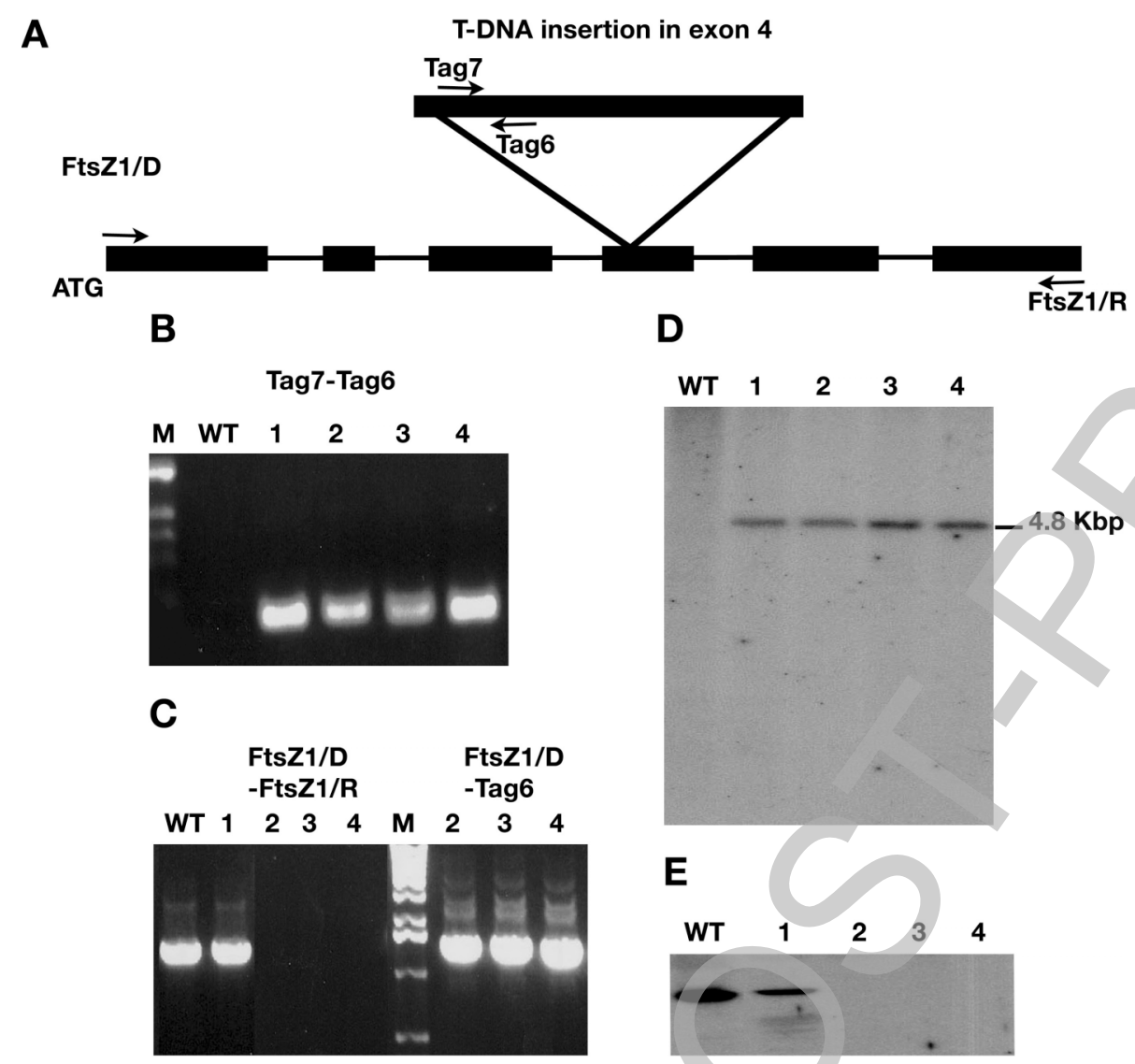

E

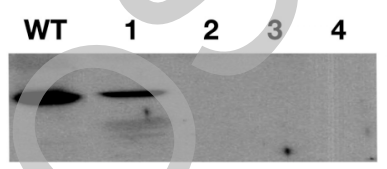

Figure 1

Licenced copy. Copying is not permitted, except with prior permission and as allowed by law. (C) 2007 The Authors Journal compilation (C) 2007 Biochemical Society 
A

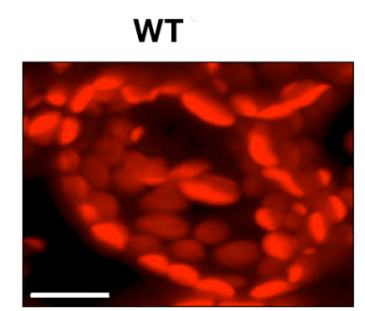

35S::FtsZ1(weak)

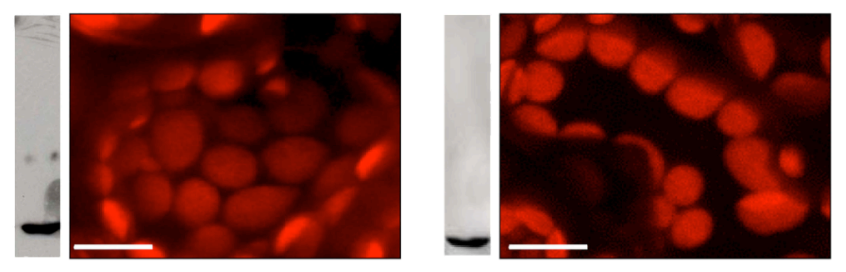

35S::FtsZ1(strong)

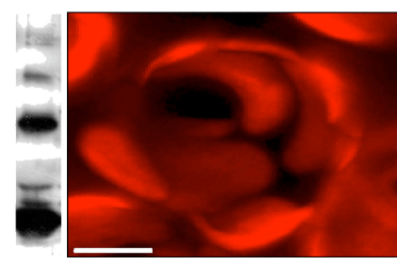

B

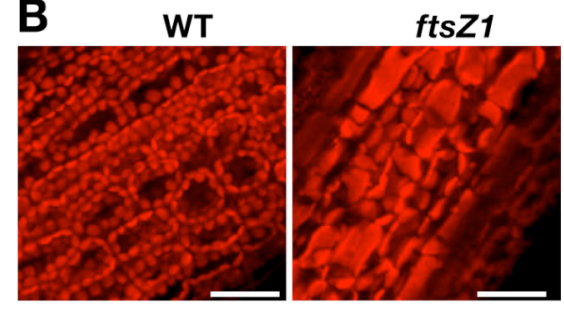

ftsZ1
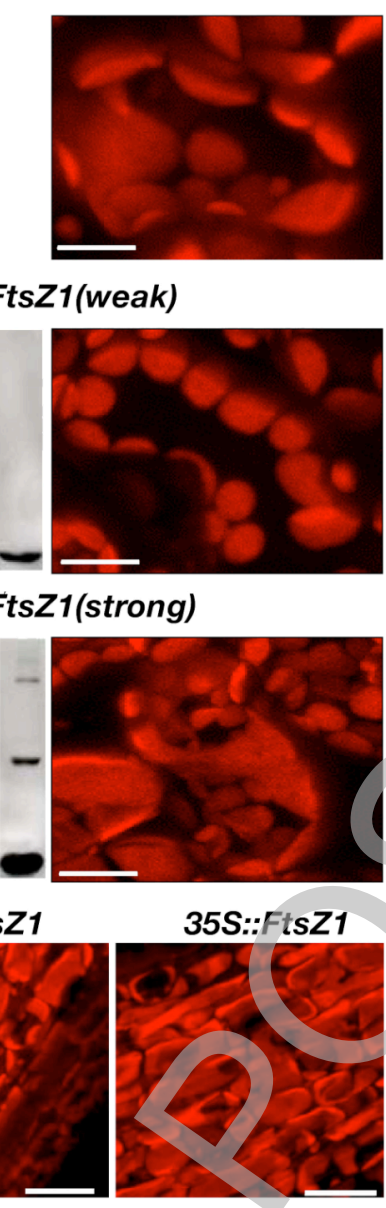

C

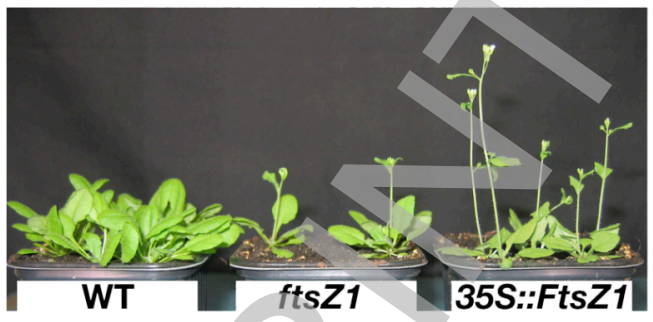

D

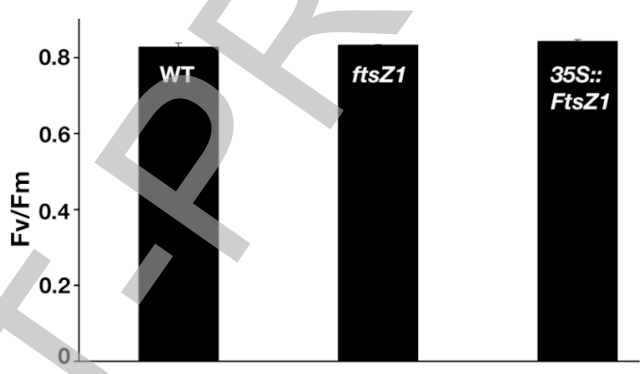

E

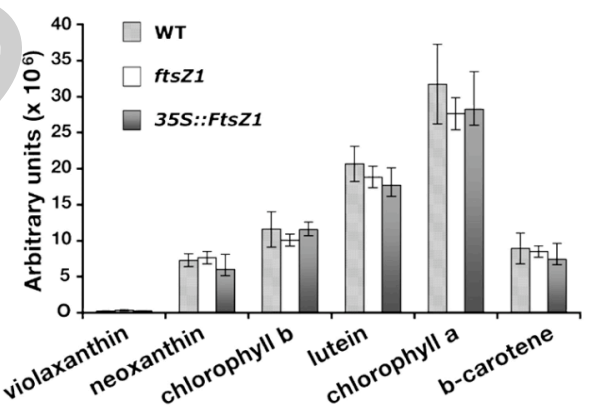

Figure 2 

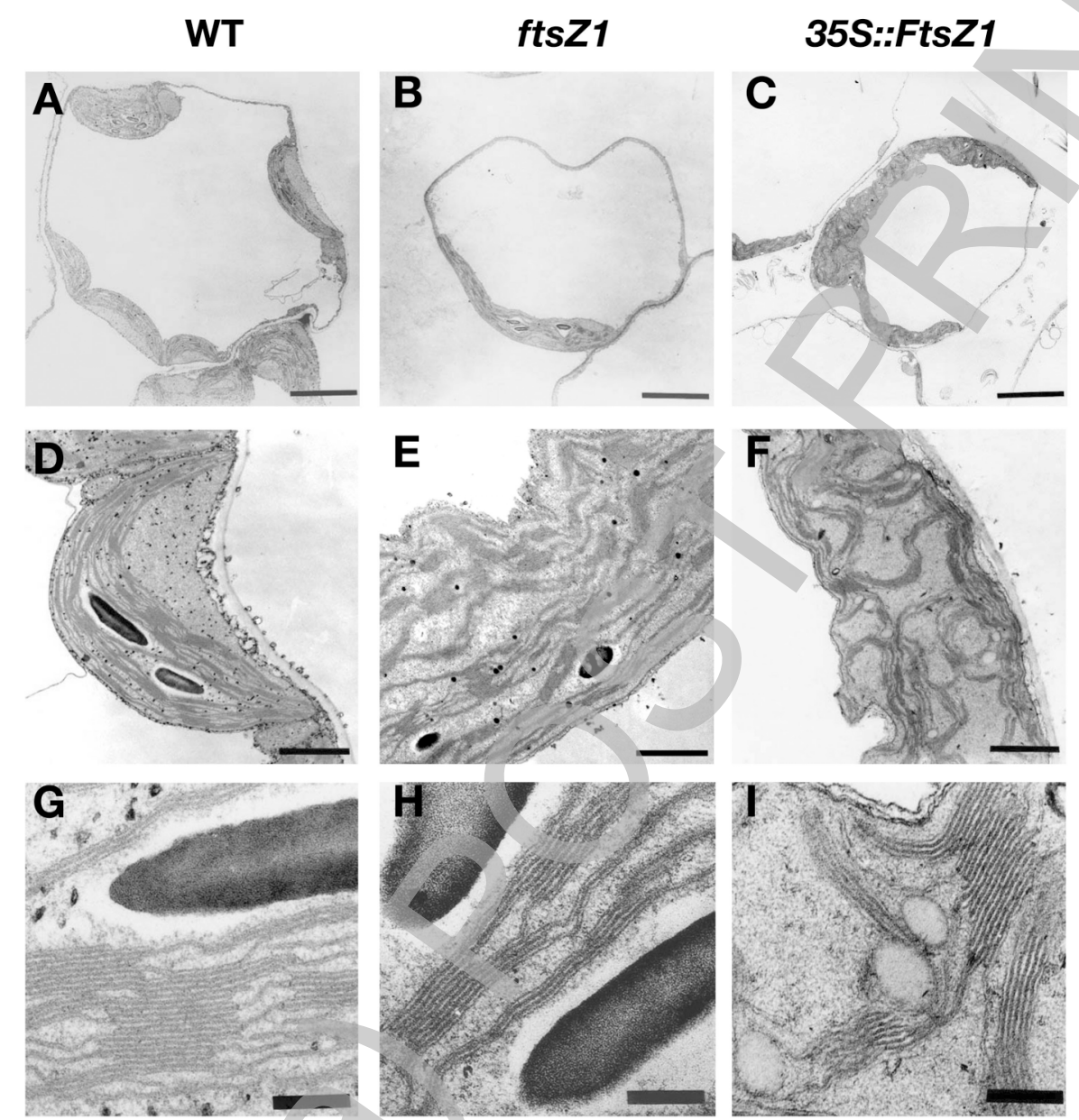

Figure 3

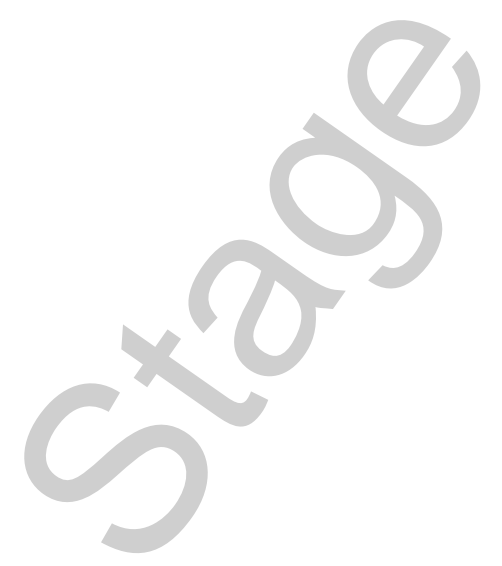

Licenced copy. Copying is not permitted, except with prior permission and as allowed by law. (C) 2007 The Authors Journal compilation (C) 2007 Biochemical Society 
A

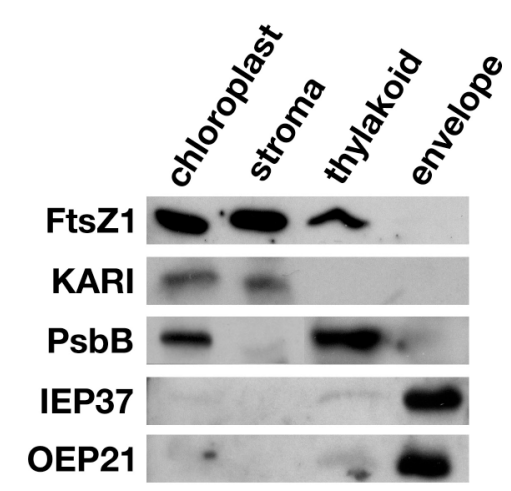

B

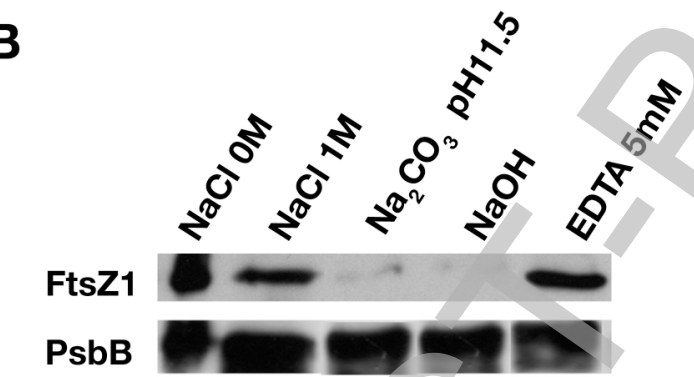

C

Figure 4

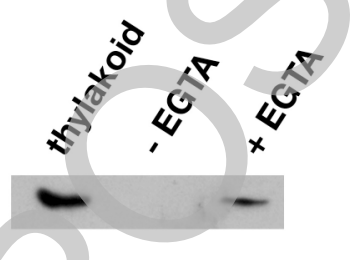

Licenced copy. Copying is not permitted, except with prior permission and as allowed by law. (C) 2007 The Authors Journal compilation (C) 2007 Biochemical Society 

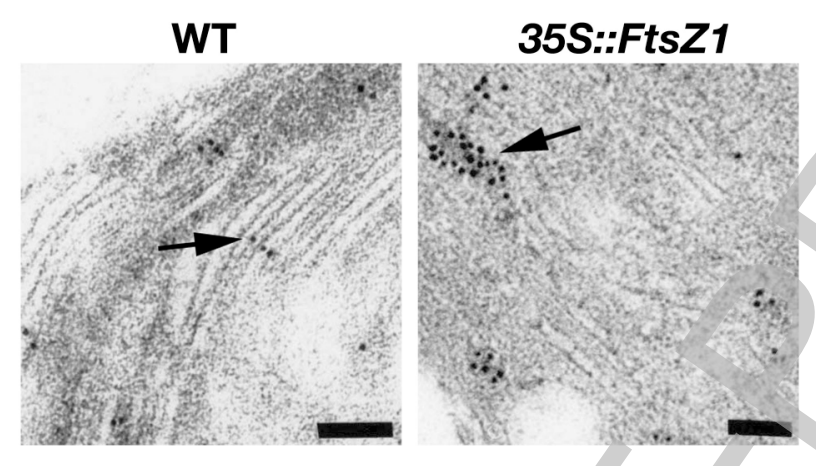

Figure 5

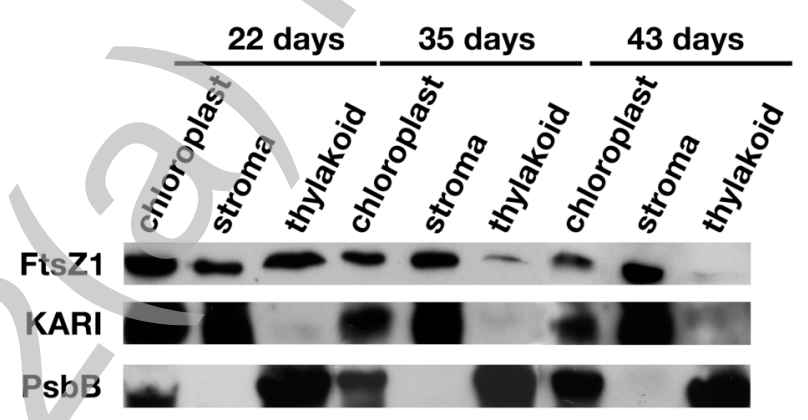

Figure 6

Licenced copy. Copying is not permitted, except with prior permission and as allowed by law. (C) 2007 The Authors Journal compilation (C) 2007 Biochemical Society 